\title{
Microstructure and Corrosion Resistance of the AlTiN Coating Deposited by Arc Ion Plating
}

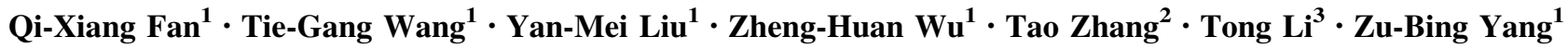

Received: 24 June 2016/Revised: 7 August 2016/Published online: 8 October 2016

(C) The Chinese Society for Metals and Springer-Verlag Berlin Heidelberg 2016

\begin{abstract}
A new type of AlTiN coating containing about 29.13 at.\% Al,16.02 at.\% Ti and 54.85 at.\% $\mathrm{N}$ was prepared by arc ion plating technology. The coating is composed of singular fcc-(Al, Ti)N phase and has no hcp-AlN phase to be formed. Due to the high content of beneficial element Al, the hardness and effective elastic modulus of the coating are up to 33.9 and $486.1 \mathrm{GPa}$, respectively. The adhesion strength between the coating and substrate is about $39.7 \mathrm{~N}$. Electrochemical test shows that the corrosion current density of the AlTiN coating is nearly one-sixth of the substrate, and the charge transfer resistance $R_{\mathrm{ct}}$ of the AlTiN coating is much larger than that of the substrate, which means that the coating could act as a protective barrier between the substrate and corrosive electrolyte, enhancing the corrosion resistance.
\end{abstract}

KEY WORDS: Arc ion plating; AITiN coating; Corrosion behavior; Microstructure; Hardness

\section{Introduction}

Nitride coatings mainly based on titanium, aluminum and chromium have been widely used as protective hard coatings in various harsh working environments owing to their high hardness and wear resistance, good thermal stability and corrosion resistance, low friction coefficient and superior chemical inertness, etc. [1]. TiN commercialized in the early

Available online at http://link.springer.com/journal/40195

Qi-Xiang Fan

qxfan2015@163.com

$\triangle$ Tie-Gang Wang

tgwang@tute.edu.cn

1 Tianjin Key Laboratory of High Speed Cutting and Precision Machining, Tianjin University of Technology and Education, Tianjin 300222, China

2 Division of State-owned Assets Management, Tianjin University of Technology and Education, Tianjin 300222, China

3 College of Electronic Engineering, Tianjin University of Technology and Education, Tianjin 300222, China 1980s is the first generation of physical vapor deposition (PVD) hard coating and is still widely used in cutting tools, dies as well as many mechanical components due to its high hardness and good wear resistance [2-5]. The oxidation resistance of TiN coating is poor since it would be oxidized quickly at the temperature above $500{ }^{\circ} \mathrm{C}$ forming a brittle and loose layer of $\mathrm{TiO}_{2}$ [6]. Compared with the TiN coatings, the $\mathrm{Ti}_{1-x} \mathrm{Al}_{x} \mathrm{~N}$ coatings possess improved corrosion resistance, oxidation resistance and thermal stability, since a dense mixed-oxide film of $\mathrm{Al}_{2} \mathrm{O}_{3}$ and $\mathrm{TiO}_{2}$ would form on the coating surface under corrosive environments or at elevated temperature which could act as a barrier hindering the diffusion of corrosive medium like $\mathrm{O}_{2}$ into the interior coating and thus improve the oxidation resistance [7, 8]. The $\mathrm{Ti}_{1-x} \mathrm{Al}_{x} \mathrm{~N}$ coatings possess face-centered cubic (fcc) structure with $\mathrm{Al}$ atoms substituting for the $\mathrm{Ti}$ atoms [7]. Because the atom radius of $\mathrm{Al}$ is smaller than that of $\mathrm{Ti}$, the lattice constant of $\mathrm{Ti}_{1-x} \mathrm{Al}_{x} \mathrm{~N}$ coatings is smaller than that of TiN coatings, and the hardness is higher. It was reported that the oxidation resistance and hardness of the nitride coatings were improved with increasing $\mathrm{Al}$ content $[9,10]$. Consequently, the AlTiN coatings with $x$ more than 0.5 are one of the promising coatings for near-dry or dry high-speed 
cutting operations [11, 12]. However, AlN with wurtzite structure (hardness $12 \mathrm{GPa}$ ) would be formed with $\mathrm{Al}$ content exceeding the maximum solubility ( $x=\mathrm{Al} /$ $(\mathrm{Al}+\mathrm{Ti})=0.65-0.75)$ in the cubic phase $[13,14]$, leading to a decrease in hardness, bulk elastic, shear moduli and wear resistance. Thus, the synthesis of single cubic AlTiN coating with high $\mathrm{Al}$ content is a challenging task.

Feng et al. [15] and $\mathrm{Xu}$ et al. [16] prepared cubic $\mathrm{Al}_{0.64} \mathrm{Ti}_{0.36} \mathrm{~N}$ coatings by cathodic arc evaporation, since the high deposition rate could increase the solubility of $\mathrm{Al}$. Their researches showed that the $\mathrm{Al}_{0.64} \mathrm{Ti}_{0.36} \mathrm{~N}$ coatings possessed good mechanical properties and oxidation resistance. Some other researchers applied multilayer architecture to increase the solubility limitation of $\mathrm{Al}$ in the cubic Ti-Al-N system by controlling the coherent growth. Todt et al. [17] prepared a kind of Al-rich cubic $\mathrm{Al}_{0.8} \mathrm{Ti}_{0.2} \mathrm{~N}$ coating with nano-lamellar microstructure by low-pressure chemical vapor deposition (PLCVD). Chen et al. [18] deposited a $\mathrm{Al}_{0.66} \mathrm{Ti}_{0.34} \mathrm{~N} / \mathrm{Ti}_{0.52} \mathrm{Al}_{0.48} \mathrm{~N}$ multilayer coating with cubic structure by cathodic arc evaporation. These multilayer coatings exhibit high hardness and good oxidation resistance because of the high Al content. Compared with single-layer coating, the preparation process of multilayer coating is difficult, and the modulation period must be very small to avoid the formation of h-AlN completely.

Hard coatings can be obtained by chemical vapor deposition (CVD) or PVD techniques such as thermal spray, ionbeam deposition, arc ion deposition and magnetron sputtering [19]. Among them, arc ion plating is one of the most widely used technologies owing to its high deposition rate and high energy of ions [16]. The coatings deposited by arc ion plating possess high density and strong adhesion. However, the macro defects such as inclusion and voids formed due to the micrometal droplets emitted from the cathodes reduce the corrosion resistance of the coatings. In many application, the coated parts are frequently exposed to an aggressive working environment, for instance, a $\mathrm{Cl}^{-}$-containing corrosive medium, particularly in a marine region, due to the strong effect of $\mathrm{Cl}^{-}$in promoting localized corrosion [2]. Thus, it is important to study the corrosion resistance of the coatings before they are applied into industry. In this paper, a new type of $\mathrm{Al}_{x} \mathrm{Ti}_{1-x} \mathrm{~N}$ coating with $x$ of $\sim 0.65$ is developed by arc ion plating technology. The microstructure, mechanical properties and corrosion resistance of the coating were investigated in detail.

\section{Experimental}

\subsection{Deposition of AITiN Coating}

The AlTiN coating was deposited on a piece of high-speed steel (HSS) by arc ion plating. The substrate with diameter of $2 \mathrm{~cm}$ was ground down to 2000 \# SiC paper and then mirror-polished to $R a<0.05 \mu \mathrm{m}$. After ultrasonically cleaned within acetone, ethanol and deionized water successively, the substrate was dried and fixed onto the rotating substrate holder at a distance of $120 \mathrm{~mm}$ from the target in the vacuum chamber. To improve the uniformity of the film, the substrate holder was rotated at a speed of $10 \mathrm{r} / \mathrm{min}$. The chamber was evacuated to at least $5 \times 10^{-4}$ $\mathrm{Pa}$ by two mechanical pumps as well as one molecular pump, and then the substrate was heated to $400{ }^{\circ} \mathrm{C}$. The elemental $\mathrm{Ti}$ and $\mathrm{Al}$ with purity of $99.99 \%$ were used as the targets. Prior to the deposition of AlTiN coating, the substrate was cleaned by Ar ion bombardment under $-700 \mathrm{~V}$ negative bias voltage for $10 \mathrm{~min}$, which would remove contaminants and enhance the adhesion strength between the film and the substrate. The current of Ti target was 50-100 A. After that, the bias voltage decreased to $60 \mathrm{~V}$ to deposit a $\mathrm{Ti}$ interlayer. This process lasted for about 10 min. Then $\mathrm{N}_{2}$ was provided, and the $\mathrm{Al}$ target was powered on. The detail deposition parameters for the AlTiN coating prepared by arc ion plating technique are listed in Table 1.

\subsection{Characterization}

Phase identifications of the AlTiN coating were carried out using the glancing X-ray diffraction (XRD, X' Pert PRD, PANalytical, Holland) to minimize the effect of high peak intensity of the high-speed steel. Surface and cross-sectional morphologies of the coating were observed under a scanning electron microscope (SEM, Inspect F) equipment with an electron-dispersive X-ray spectrometer (EDS) for compositional analysis. The surface roughness of the coating was measured by using a surface profiler (AlphaStep IQ, KLA Tencor).

The mechanical properties of the AlTiN coating were measured by using a nano-indentation tester (Nano

Table 1 Deposition parameters of the AlTiN coating by arc ion plating technology

\begin{tabular}{ll}
\hline Parameters & Value \\
\hline Base pressure (Pa) & $5 \times 10^{-4}$ \\
Working pressure (Pa) & 2 \\
Bias voltage (V) & -60 \\
Ar flow (sccm) & $50-100$ \\
$\mathrm{~N}_{2}$ flow (sccm) & $300-400$ \\
Deposition temperature $\left({ }^{\circ} \mathrm{C}\right)$ & 400 \\
Deposition time (min) & 90 \\
Arc current of Ti target (A) & $50-100$ \\
Arc current of Al target (A) & $50-100$ \\
Distance between the substrate and target $(\mathrm{mm})$ & 120 \\
\hline
\end{tabular}


indenter G200, Agilent Technologies, USA) with a Berkovich indenter tip under the continuous stiffness mode. Ten separated measurements were taken on the AlTiN coating to obtain the average value. The distance between each indentations was at least 30 times of the maximum depth of indentation to prevent stress field effects from nearby indentations. The maximum depth of indentation was set as $1500 \mathrm{~nm}$. The calibration of the film hardness values was checked by measuring on a standard sample (fused silica). The hardness and elastic modulus of the sample were $8.86 \pm 0.11$ and $72.1 \pm 0.3 \mathrm{GPa}$, respectively. To avoid the effect of the substrate and get hardness $(H)$ as well as effective elastic modulus $\left(E^{*}\right)$ more accurately, the measured values are chosen at the indentation depth less than $10 \%$ of the film thickness. Here, $E^{*}$ is given directly by the nano-indentation tester based on the formula $E^{*}=E /\left(1-v^{2}\right)$, where $E$ is the elastic modulus and $v$ is the Poisson's ratio.

The adhesion strength between the coating and substrate was measured by a scratch tester (J\&L Tech, Scratch test JLST022) with a Rockwell C diamond stylus (cone apex angle: $120^{\circ}$; tip radius: $200 \mu \mathrm{m}$ ) that was moved across the sample surface. During the scratch tests, the normal load was increased from 0 to $100 \mathrm{~N}$ with a loading rate of $1 \mathrm{~N} / \mathrm{s}$ and a translation speed of $0.2 \mathrm{~mm} / \mathrm{s}$. The friction force, friction coefficient, and acoustic emission signals were also recorded during the scratch tests in order to measure the critical load. The scratch tracks were observed under an optical microscope (OM, Olympus PMG3-613U W/Acc).

The electrochemical measurements of the AlTiN coating were taken in a $3.5 \mathrm{wt} \% \mathrm{NaCl}$ solution, since chloride ioncontaining environments are very common. The electrochemical properties of the AlTiN coating were tested by Gamry Instruments (USA) with an active area of $1 \mathrm{~cm}^{2}$. A three-electrode system was used, consisting of a saturated calomel electrode (SCE) as a reference, a pure platinum sheet as counter electrode and the coated sample under test as the working electrode. The electrolyte employed was $3.5 \mathrm{wt} \%$ sodium chloride solution, prepared with analytical grease reagents and deionized water. Before the electrochemical measurements, samples were allowed to stabilize at their open-circuit potential $\left(E_{\mathrm{oc}}\right)$ for $30 \mathrm{~min}$. The potentiodynamic polarization measurements were taken starting from $-200 \mathrm{mV}$ cathodically with respect to the open-circuit potential at a constant scan rate of $0.5 \mathrm{mV} / \mathrm{s}$. The electrochemical impedance spectroscopy (EIS) measurement was taken using a scanning frequency ranging from $10^{-2}$ to $10^{5} \mathrm{~Hz}$, and the testing signal is $20 \mathrm{mV}$. All the measurements are taken at room temperature $\left(22^{\circ} \mathrm{C}\right)$. Based on an appropriate electrical equivalent circuit, the Nyquist plots obtained from the EIS measurements were fitted by ZSimpwin 3.20 software to calculate the corresponding parameters.

\section{Results and Discussion}

\subsection{Microstructure Characterization}

Figure 1 shows the XRD patterns of the AlTiN coating deposited by arc ion plating. As can be seen, the coating exhibits a single fcc structure with multiple orientations of (111), (200), (220), (311) and (222) crystal planes. The diffraction peaks lie between those of fcc TiN (PDF\# 25-1495) and AlN (PDF\# 38-1420) phases, indicating that the fcc (Al, Ti)N solid solution phase forms, while the B4wurtzite AlN (PDF\# 25-133) does not form. The AlTiN coating contains higher $\mathrm{Al}$ content than Ti content; therefore, the diffraction peaks of the coating are closer to those of AlN which possesses smaller lattice constant than TiN. The preferred orientation of the AlTiN coating is (111) crystal plane which has the lowest surface energy in the fcc structure [20]. The preferred orientation is usually the crystal plane which possesses the minimum overall energy between surface energy and strain energy during the deposition process $[21,22]$. Since the strain energy of (200) plane is smaller than that of the (111) plane [23], the preferred orientation would be (200) crystal plane when the deposition parameters such as deposition temperature and bias voltage change [4, 24].

Figure 2 shows the surface morphologies of the AlTiN coating deposited by arc ion plating. As shown in Fig. 2, there are some white particles and pinholes on the dark gray surface. The white particles are macro-particles resulting from the droplets which were emitted from the arc spots in the $\mathrm{Al}$ target or Ti target and solidified before they arrived at the substrate. The macro-particles shrink at the ambient temperature after deposition. The macro-particles possess larger specific area than the coating around them,

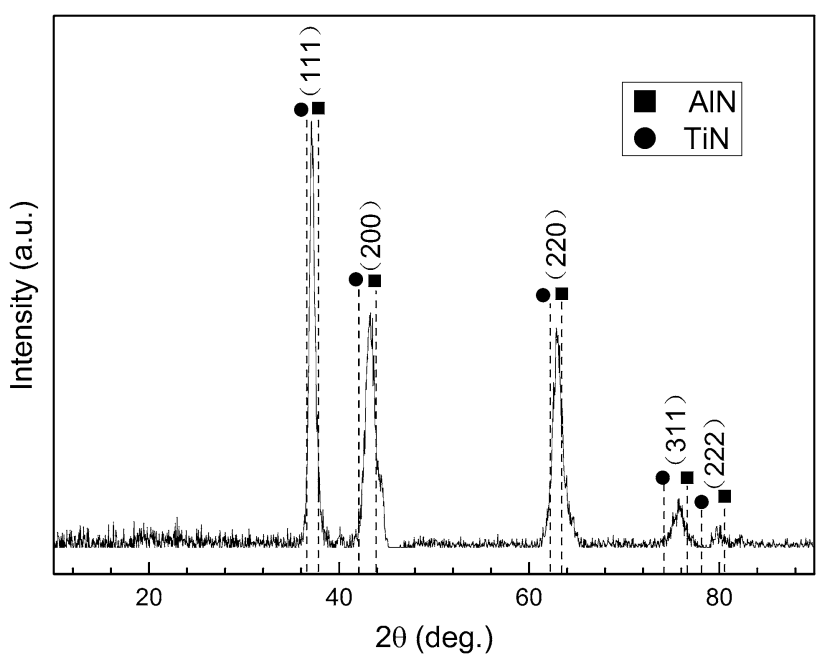

Fig. 1 XRD patterns of the AlTiN coating deposited by arc ion plating technology 


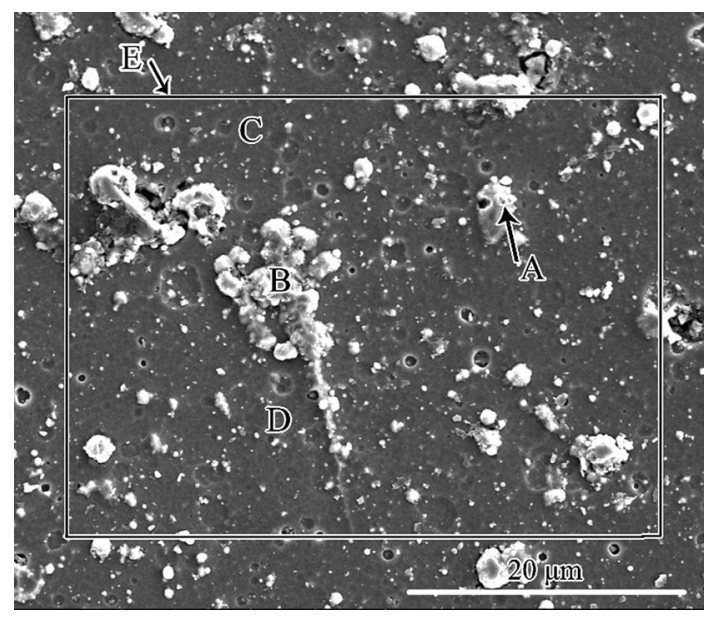

Fig. 2 Surface morphologies of the AlTiN coating deposited by arc ion plating technology

Table 2 Elemental compositions of four different points (A, B, C, D) and one selected area $(\mathrm{E})$ on the surface of the AlTiN coating

\begin{tabular}{lllll}
\hline Position & $\mathrm{Al}($ at.\%) & $\mathrm{Ti}($ at.\%) & $\mathrm{N}($ at.\%) & $\mathrm{Al} /(\mathrm{Al}+\mathrm{Ti})(x)$ \\
\hline $\mathrm{A}$ & 28.35 & 15.37 & 56.26 & 0.648 \\
$\mathrm{~B}$ & 25.20 & 15.13 & 59.68 & 0.625 \\
$\mathrm{C}$ & 32.71 & 18.87 & 48.42 & 0.634 \\
$\mathrm{D}$ & 29.80 & 15.04 & 55.16 & 0.665 \\
$\mathrm{E}$ & 29.13 & 16.02 & 54.85 & 0.645 \\
\hline
\end{tabular}

thereby they shrink more obviously, and some might flake from the surface resulting in the pits and pinholes left on the coatings [25, 26]. Because of these macro-particles and pinholes, the surface roughness of the coating is high (about $214.3 \mathrm{~nm}$ ) measured by surface profiler.

The elemental compositions at four different points (A, B, C, D in Fig. 2) and in one selected area (inside area of the rectangle $E$ in Fig. 2) on the surface of the AlTiN coating are analyzed by EDS, which is listed in Table 2. As can be seen, the white macro-particles contain more $\mathrm{N}$ element than the dark gray spots, and the $\mathrm{Al} /(\mathrm{Al}+\mathrm{Ti})$ values range from 0.625 to 0.665 . The elemental compositions in the rectangle $\mathrm{E}$ is about 29.13 at.\% $\mathrm{Al}$, 16.02 at. $\% \mathrm{Ti}$ and 54.85 at. $\% \mathrm{~N}$. The $\mathrm{Al} /(\mathrm{Al}+\mathrm{Ti})$ value is about 0.65 . Since the selected area $\mathrm{E}$ includes the four points (A, B, C, D), the elemental compositions of this area $\mathrm{E}$ are taken as the average elemental compositions of the coating. The cross-sectional morphology of the AlTiN coating is presented in Fig. 3. It can be seen that a very thin layer of Ti lies between the AlTiN coating and the substrate, which plays an important role in the improvement of adhesive strength. The AlTiN coating is dense, and the thickness is about $3.0 \mu \mathrm{m}$.

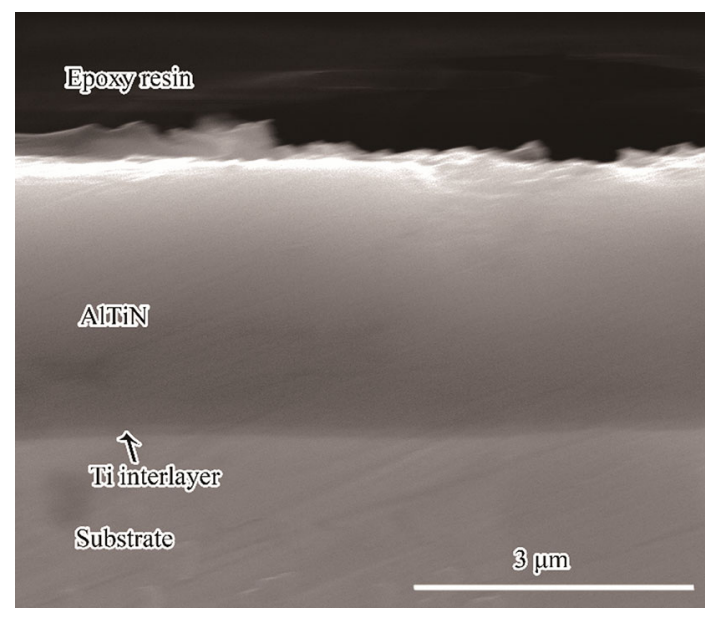

Fig. 3 Cross-sectional morphology of the AlTiN coating deposited by arc ion plating technology

\subsection{Mechanical Properties}

The hardness versus displacement and modulus versus displacement curves of the AlTiN coating are presented in Fig. 4. These two curves show the same trend which could be divided into three stages: quickly increasing stage, plateau stage and declining stage. The average hardness value at the plateau stage could be considered as the real hardness of the films. Note that the indentation depth at this region is less than $10 \%$ of the film thickness, which could be deemed to minimize the effect of the substrate greatly. The hardness and effective modulus $\left(E^{*}\right)$ of the AlTiN coating are about 33.94 and $486.1 \mathrm{GPa}$, respectively. The high hardness of the coating is attributed to the solid solution hardening of the element $\mathrm{Al}$ which decreases the lattice constant of the coating. It is consistent with the XRD pattern (Fig. 1) that the diffraction peaks of the AlTiN coating shift to higher angles, compared with those of TiN phase.

The adhesion strength of the AlTiN coating was measured by scratching its surface with the normal load increasing gradually from 0 to $100 \mathrm{~N}$. During the process, plastic deformation occurs at the beginning; then, some cracks form and flakes might be generated along the track subsequently. Finally, large spallation outside the track happens and makes the coating detach from the substrate indicating the total failure of the film [27]. Figure 5 shows the morphology of the scratch track of the AlTiN coating. When the normal force is up to about $39.7 \mathrm{~N}$, large spallation occurs and the coating is removed from the substrate. The adhesion strength of a coating/substrate system is normally defined as the energy to separate a coating from the substrate [28]. Thus, the adhesion strength of the AlTiN coating is about $39.7 \mathrm{~N}$. 

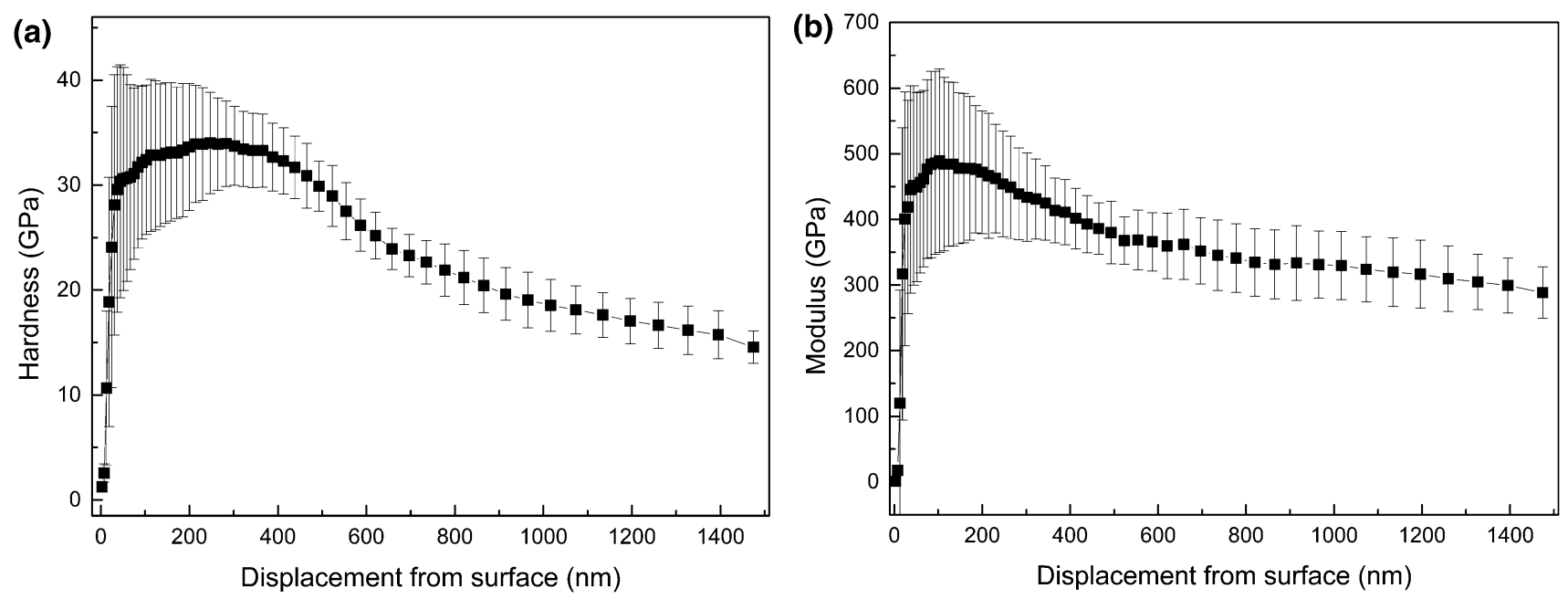

Fig. 4 Hardness versus displacement $\mathbf{a}$ and modulus versus displacement and $\mathbf{b}$ of the AlTiN coating measured by nano-indenter tester

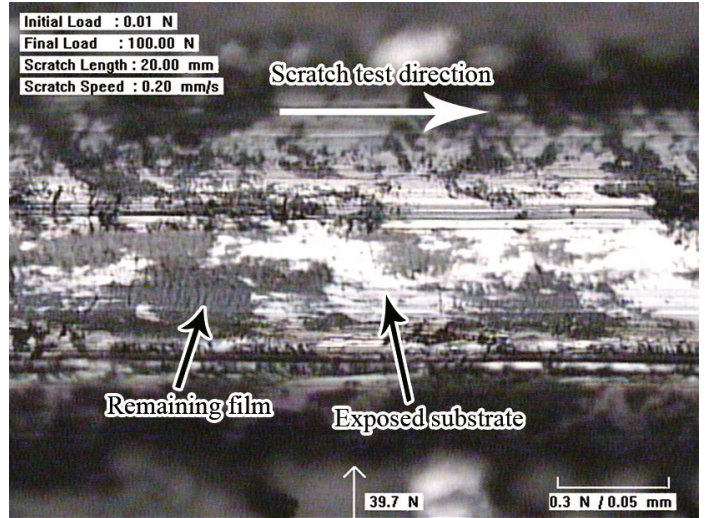

Fig. 5 Morphology of scratch track of the AlTiN coating observed by optical microscopy

\subsection{Electrochemical Properties}

The potentiodynamic polarization curves of the substrate and AlTiN coating in a $3.5 \mathrm{wt} \% \mathrm{NaCl}$ solution are shown in Fig. 6. The corrosion potential $E_{\text {corr }}$ and current density $I_{\text {corr }}$ of the substrate are about $-0.5019 \mathrm{~V}$ and $11.392 \mu \mathrm{A} /$ $\mathrm{cm}^{2}$, respectively, while for the AlTiN coating the corrosion potential is $-0.4999 \mathrm{~V}$ and the current density is $1.861 \mu \mathrm{A} / \mathrm{cm}^{2}$ according to the Tafel extrapolation method. The potential of AlTiN coating is slightly lower than that of the substrate, owing to the high electrochemical activity of $\mathrm{Al}$ and $\mathrm{Ti}$. However, the corrosion current density of the AlTiN coating is nearly one-sixth of that of the substrate. The value of the corrosion potential reflects the corrosion tendency of the materials in thermodynamics, and the value of the corrosion current density reflects the corrosion rate of the material in kinetics [29]. Usually, $I_{\text {corr }}$ is cited as a criterion to evaluate the kinetics of a corrosion process, and

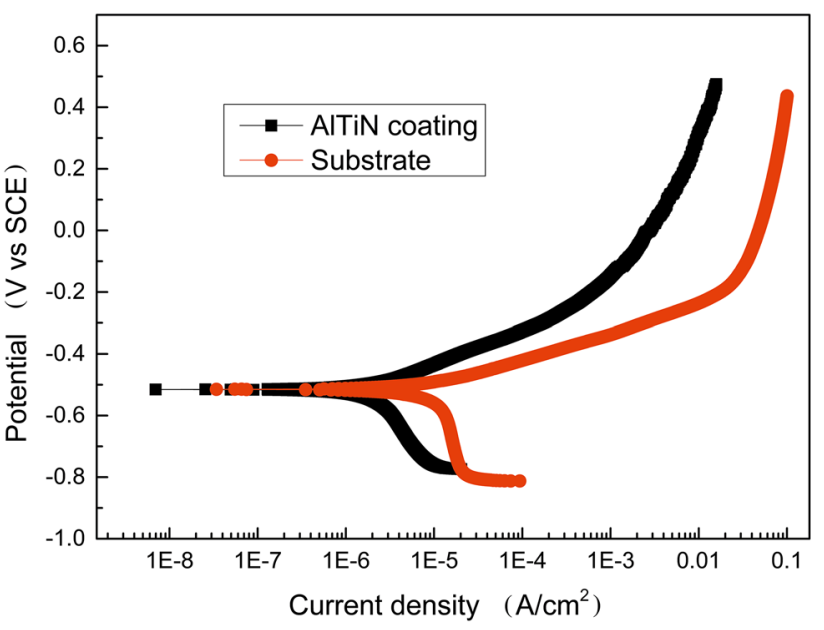

Fig. 6 Potentiodynamic polarization curves of the substrate and AlTiN coating in $3.5 \mathrm{wt} \% \mathrm{NaCl}$ solution

it is normally proportional to the corrosion rate [1]. The corrosion current density of the AlTiN coating is lower than that of the substrate meaning that it possesses better corrosion resistance than the substrate. The nitride coatings could form thin passive oxide layers on the coatings' surface when the coating samples are contacting with the $\mathrm{NaCl}$ solution [2]. Since the AlTiN coating contains high $\mathrm{Al}$ content about 30.58 at.\%, and the Gibbs free energy of $\mathrm{Al}_{2} \mathrm{O}_{3}$ is lower than that of $\mathrm{TiO}_{2}$, a continuous and dense oxide layer mainly composed of alumina could be formed on the surface of the coating during the corrosion process $[30,31]$. The oxide layer can act as a protective barrier between the substrate and the corrosive electrolyte, enhancing the corrosion resistance greatly.

The EIS of the substrate and AlTiN coating in $3.5 \mathrm{wt} \%$ $\mathrm{NaCl}$ solution is illustrated in Fig. 7. The EIS of the 


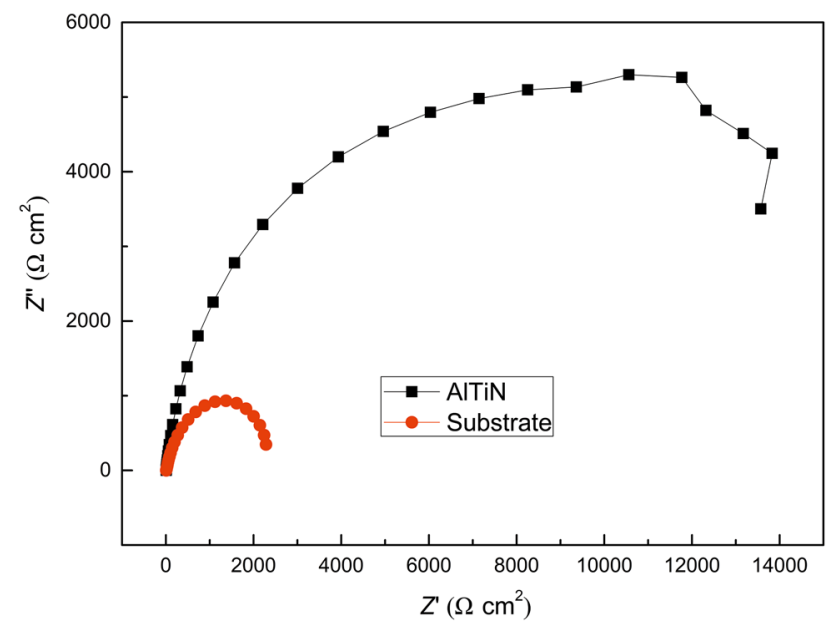

Fig. 7 Electrochemical impedance spectroscopy (EIS) of the substrate and AlTiN coating in $3.5 \mathrm{wt} \% \mathrm{NaCl}$ solution

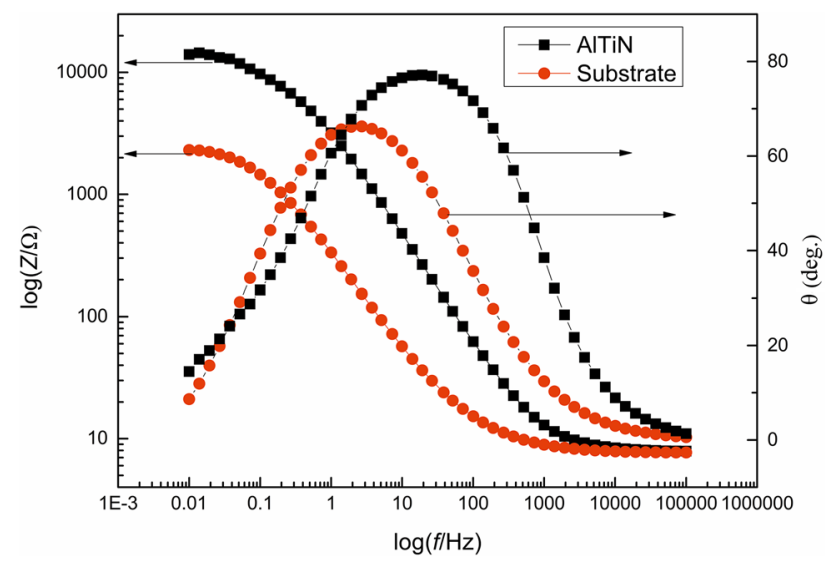

Fig. 8 Bode and phase diagram of the substrate and AlTiN coating in $3.5 \mathrm{wt} \% \mathrm{NaCl}$ solution

substrate and the AlTiN coating shows an irregular semicircle indicating a single time constant, for the samples were exposed in the corrosive medium for just approximately $1 \mathrm{~h}$, which is not sufficient to reveal the degradation of the substrate. Usually, the larger the diameter of the capacitive loop, the better the corrosion resistance of the sample [32]. The AlTiN coating possesses a larger capacitive resistance than the substrate, indicating that the AlTiN coating has better corrosion resistance. As shown in Fig. 8, the absolute impedance of the AlTiN coating is much larger than that of the substrate, which also indicates that the AlTiN coating possesses superior corrosion resistance to the substrate.

After ZsimpWin 3.20 software fitting, the EIS data of the substrate and AlTiN coating can be fit well with the equivalent circuit in Fig. 9a, b, respectively. In the equivalent circuit, the electrolyte resistance $R_{\mathrm{s}}$ is electrolyte solution resistance between the working electrode and the
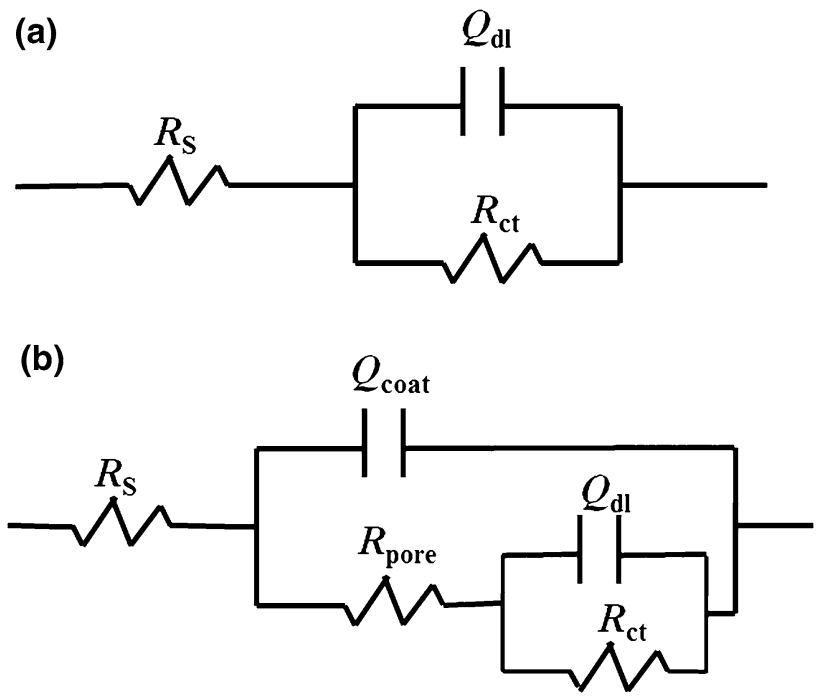

Fig. 9 Equivalent electrical circuit for the EIS data of the substrate a and AlTiN coating b corroded in $3.5 \mathrm{wt} \% \mathrm{NaCl}$ solution

reference electrode. $Q_{\mathrm{dl}}$ is a double-layer capacitance related to the electrochemical process occurring between the alloy and the solution, while $R_{\mathrm{ct}}$ represents the charge transfer process happening between the alloy and the electrolyte. For the AlTiN coating, the equivalent circuit contains two additional elements, the capacitance $Q_{\text {coat }}$ and the charge transfer resistance $R_{\text {pore }}$ resulting from the formation of ionic conduction path across the coating. The corrosion interface is characterized by a parallel capacitor with a double-layer constant phase element $Q_{\mathrm{d} 1}$ and charge transfer resistance $R_{\mathrm{ct}}$. Note that $Q$ stands for constant phase element, which is responsible for deviation from ideal dielectric behavior of a non-ideal capacitor. The admittance is given by:

$Y(\omega)=Y_{0}(j \omega)^{n}$,

where $Y_{0}$ is the adjustable parameter used in the nonlinear least squares fitting, $j$ is $\sqrt{-1}, \omega$ is the angular frequency, and $n$ is also an adjustable parameter which always lies in -1 and 1 . For $n=1, Y$ represents a capacitance, while for $n<1, Q$ is a non-ideal capacitor. For $n=-1, Y$ represents an inductance; for $n=0, Y$ represents a resistance.

The parameters extracted from the EIS data of the bare alloy and AlTiN coating corroded in $3.5 \mathrm{wt} \% \mathrm{NaCl}$ solution is presented in Table 3. The charge transfer resistance $R_{\mathrm{ct}}$ of the AlTiN coating is about $1.22 \times 10^{4} \Omega \mathrm{cm}^{2}$, which is much larger than that of the substrate $\left(2633 \Omega \mathrm{cm}^{2}\right)$. Usually, the higher the charge transfer resistance, the better the corrosion resistance of the material [33]. In addition, the double-layer capacitance of the AlTiN coating is $2.93 \times 10^{-4} \mu \mathrm{F} / \mathrm{cm}^{2}$, smaller than that of the substrate $\left(6.358 \times 10^{-4} \mu \mathrm{F} / \mathrm{cm}^{2}\right)$, which indicates that the number of electric charge and ion at the corrosive interface is less, 
Table 3 Parameters extracted from the EIS data of the substrate and AlTiN coating corroded in $3.5 \mathrm{wt} \% \mathrm{NaCl}$ solution

\begin{tabular}{llllllll}
\hline Sample & $R_{\mathrm{s}}\left(\Omega \mathrm{cm}^{2}\right)$ & $Q_{\text {coat }}-Y_{\mathrm{o}}\left(\mu \mathrm{F} / \mathrm{cm}^{2}\right)$ & $n_{\mathrm{c}}$ & $R_{\text {pore }}\left(\Omega \mathrm{cm}^{2}\right)$ & $Q_{\mathrm{dl}}-Y_{\mathrm{o}}\left(\mu \mathrm{F} / \mathrm{cm}^{2}\right)$ & $n_{\mathrm{dl}}$ & $R_{\mathrm{ct}}\left(\Omega \mathrm{cm}^{2}\right)$ \\
\hline Substrate & 8.046 & - & - & - & $6.358 \times 10^{-4}$ & 0.7779 & 2633 \\
AlTiN & 8.68 & $5.43 \times 10^{-5}$ & 0.8833 & 8536 & $2.93 \times 10^{-4}$ & 0.612 & $1.22 \times 10^{4}$ \\
\hline
\end{tabular}

revealing that the AlTiN film is corroded milder than the bare alloy. It is consistent with the potentiodynamic polarization result. Thus, it can be concluded that the coating can act as a protective barrier between the substrate and the corrosive electrolyte and enhance corrosion resistance effectively regardless of the presence of macro-particles and pinholes on the surface.

\subsection{Porosity of Coating}

The porosity of a coating has a great impact on the properties of the coating. If the coatings possess high porosity, severe corrosion between the coating and substrate would occur, since the substrate would take as the anode. Several electrochemical methods could estimate the porosity of a coating [34]. For nitride coatings, the porosity of a coating can be determined from the polarization resistance measurements according to Eq. (2) [1, 34]:

$P=\left(\frac{R_{\mathrm{ps}}}{R_{\mathrm{p}}}\right) \times 10^{-\left|\Delta E_{\mathrm{corr}}\right| / \beta_{\mathrm{a}}}$,

where, $P$ is the total coating porosity; $R_{\mathrm{ps}}$ and $R_{\mathrm{p}}$ are the polarization resistance of the substrate and the coating, respectively; $\Delta E_{\text {corr }}$ is the difference between the corrosion potential of the coating and the substrate; $\beta_{\mathrm{a}}$ is the anodic Tafel slope of the substrate.

According to Table $3, R_{\mathrm{ps}}$ is equal to $2633 \Omega \mathrm{cm}^{2}$, and $R_{\mathrm{p}}$ is $2.0556 \times 10^{4} \Omega \mathrm{cm}^{2}$ (the combination of $R_{\text {pore }}$ and $R_{\mathrm{ct}}$ ). $\Delta E_{\text {corr }}$ is about $0.002 \mathrm{~V}$, and $\beta_{\mathrm{a}}$ is $0.1074 \mathrm{~V} /$ decade by linear fitting the anode polarization curve. Putting these parameters in formula (2), the porosity can be calculated out as $12.27 \%$.

\section{Conclusions}

1. A new type of AlTiN coating with $\mathrm{Al} /(\mathrm{Al}+\mathrm{Ti})$ about 0.65 was prepared on a high-speed steel by arc ion plating technology. The coating consists of singular fcc-(Al, Ti)N phase with preferred orientation (111) crystal plane.

2. The hardness and the elastic modulus of the AlTiN coating are about 33.94 and $486.1 \mathrm{GPa}$, respectively. The adhesion strength between the coating and substrate is $39.7 \mathrm{~N}$.
3. The AlTiN coating possesses lower corrosion current density and larger charge transfer resistance than the bare alloy, which indicates that the coating has better corrosion resistance in $3.5 \mathrm{wt} \% \mathrm{NaCl}$ electrolyte and could act as a protective barrier between the substrate and corrosive electrolyte.

Acknowledgments This work was supported by the National Nature Science Foundation of China (Grant Nos. 51501130 and 51301181), the Tianjin Key Research Program of Application Foundation and Advanced Technology (Grant No. 15JCZDJC39700), the Innovation Team Training Plan of Tianjin Universities and colleges (Grant No. TD12-5043), the Tianjin Science and Technology correspondent project (16JCTPJC49500), and Research Development Foundation of Tianjin University of Technology and Education (Grant No. KJ1422).

\section{References}

[1] L. Zhang, Y. Chen, Y. Feng, S. Chen, Q. Wan, J. Zhu, Int. J. Refract. Metals Hard Mater. 53, 68 (2015)

[2] X.Z. Ding, A.L.K. Tan, X.T. Zeng, C. Wang, T. Yue, C.Q. Sun, Thin Solid Films 516, 5716 (2008)

[3] Z. Wang, D. Zhang, P. Ke, X. Liu, A. Wang, J. Mater. Sci. Technol. 31, 37 (2015)

[4] Y.H. Zhao, W.J. Yang, C.Q. Guo, Y.Q. Chen, B.H. Yu, J.Q. Xiao, Acta Metall. Sin. (Engl. Lett.) 28, 10 (2015)

[5] X. Chen, J. Zhang, S. Ma, H. Hu, Z. Zhou, Acta Metall. Sin. (Engl. Lett.) 27, 31 (2014)

[6] D. McIntyre, J. Greene, G. Håkansson, J.E. Sundgren, W.D. Münz, J. Appl. Phys. 67, 1542 (1990)

[7] D. Kong, H. Guo, Tribol. Int. 88, 31 (2015)

[8] D. Kong, G. Fu, J. Mater. Res. 30, 503 (2015)

[9] J. Peng, D. Su, C. Wang, J. Mater. Sci. Technol. 30, 803 (2014)

[10] T. Polcar, A. Cavaleiro, Mater. Chem. Phys. 129, 195 (2011)

[11] M.I. Yousaf, V.O. Pelenovich, B. Yang, C.S. Liu, D.J. Fu, Surf. Coat. Technol. 265, 117 (2015)

[12] X. Wang, P.Y. Kwon, D. Schrock, D. Kim, Wear 304, 67 (2013)

[13] L. Chen, M. Moser, Y. Du, P.H. Mayrhofer, Thin Solid Films 517, 6635 (2009)

[14] Y. Makino, K. Nogi, Surf. Coat. Technol. 98, 1008 (1998)

[15] Y. Feng, L. Zhang, R. Ke, Q. Wan, Z. Wang, Z. Lu, Int. J. Refract. Metals Hard Mater. 43, 241 (2014)

[16] Y. Xu, K. Chen, S. Wang, C. Pan, S. Chen, Vacuum 131, 97 (2016)

[17] J. Todt, J. Zalesal, R. Daniel, R. Pitonak, A. Köpf, R. Weißenbacher, B. Sartory, C. Mitterer, J. Keckes, Surf. Coat. Technol. 291, 89 (2016)

[18] L. Chen, Y. Xu, Y. Du, Y. Liu, Thin Solid Films 592, 207 (2015)

[19] V.K.W. Grips, H.C. Barshilia, V.E. Selvi, Kalavati, K.S. Rajam, Thin Solid Films 514, 204 (2006)

[20] H. Jiménez, E. Restrepo, A. Devia, Surf. Coat. Technol. 201, 1594 (2006) 
[21] P.J. Martin, A. Bendavid, T.J. Kinder, I.E.E.E. Trans, Plasma Sci. 25, 264 (1997)

[22] G.Q. Yu, B.K. Tay, S.P. Lau, K. Prasad, L.K. Pan, J.W. Chai, D. Lai, Chem. Phys. Lett. 374, 264 (2003)

[23] J. Pelleg, L.Z. Zevin, S. Lungo, N. Croitoru, Thin Solid Films 197, 117 (1991)

[24] F. Cai, S. Zhang, J. Li, Z. Chen, M. Li, L. Wang, Appl. Surf. Sci. 258, 1819 (2011)

[25] C.M. Shi, T.G. Wang, Z.L. Pei, J. Gong, C. Sun, J. Mater. Sci. Technol. 30, 1193 (2014)

[26] T.G. Wang, D. Jeong, S.H. Kim, Q. Wang, D.W. Shin, S. Melin, S. Iyengar, K.H. Kim, Surf. Coat. Technol. 206, 2629 (2012)

[27] Y.M. Liu, C.L. Jiang, Z.L. Pei, H. Lei, J. Gong, C. Sun, Surf. Coat. Technol. 245, 108 (2014)

[28] T.G. Wang, D. Jeong, Y. Liu, Q. Wang, S. Iyengar, S. Melin, K.H. Kim, Surf. Coat. Technol. 206, 2638 (2012)
[29] N. Perez, Electrochemical and Corrosion Science (Chemical Insustry Press, Beijing, 2013), p. 54. (in Chinese)

[30] Y.J. Liang (ed.), Handbook of Thermodynamics Data for Inorganic Coumpounds (Northeastern University Press, Shenyang, 1993)

[31] D.L. Wu, S.M. Jiang, Q.X. Fan, J. Gong, C. Sun, Acta Metall. Sin. (Engl. Lett.) 27, 627 (2014)

[32] L.M. Chang, D. Chen, S.Y. Shi, Mater. Prot. 41, 17 (2008). (in Chinese)

[33] Y. Li, L. Qu, F.H. Wang, Z.B. Shao, J. Chin. Soc. Corros. Prot. 23, 65 (2003). (in Chinese)

[34] J. Creus, H. Mazille, H. Idrissi, Surf. Coat. Technol. 130, 224 (2000) 